\title{
Emergency Management in PHASE I Studies in Italy. Rationale and Operating Procedures
}

\section{Daniela Maria Capuano and Roberto Verna*}

Department of Experimental Medicine, World Association of Societies of Pathology and Laboratory Medicine, Sapienza University of Rome, Italy

*Corresponding Author: Roberto Verna, Department of Experimental Medicine, World Association of Societies of Pathology and Laboratory Medicine, Sapienza University of Rome, Italy.

Received: November 15, 2019; Published: November 29, 2019

DOI: $10.31080 /$ ASPS.2019.03.0448

\section{Abstract}

A clinical trial is a scientific study that aims to verify the efficacy of an experimental drug for diseases for which there is no adequate cure or to show that a new drug is better than the one already used for the same disease.

Keywords: Phase I; Italy; Drug

The Good Clinical Practices (GCPs) guarantee the protection of the rights, safety and well-being of the participants in clinical trials and the reliability and accuracy of the data obtained [1,2].

In developing a new drug, a clinical trial on man proceeds by degrees and is divided into four Phases: Clinical Phases I - II - III -IV. The first three Phases are for registration purposes and range from the first administration to humans until they are placed on the market; Phase IV refers to marketing and post-marketing surveillance or pharmacovigilance.

Phase I

Purely cognitive and non-therapeutic Phase that aims to assess the tolerability and safety of the new drug:

- Pharmacokinetic and pharmacodynamic studies are carried out.

- Conducted on a limited number of healthy volunteers and/ or patients (in oncology) who are not responsive to standard treatments.

- With single and repeated administrations.

- $\quad$ The maximum tolerated dose (DMT) beyond which adverse events occur is defined.
As to the Phase I studies, the Italian Agency for Medicines (AIFA), in 2015 issued the Determination 809 [3] with which it establishes the requirements that the clinical centers that perform clinical trials of Phase I must possess in terms of structures, equipment, personnel, emergency management and quality requirements that need the establishment of written Standard Operative Protocols (SOPs).

In 2016, with the determination 451 [4], the AIFA establishes the methods with which the Phase I structures must notify the possession of the required requirements. All this takes place through a self-certification process sent at least 90 days before the start of the activities by certified mail. On the basis of the self-certifications and the outcome of any verifications, made by the Office of Inspection Activities GCP, the AIFA publishes, through the Observatory of Clinical trials (OsSc), the lists of the accredited centers, divided by Region, for the Phase I trials [5].

The Determination unfolds in 6 articles of which article 2 reports the requirements that must be in possession and which are detailed in Annex 1 and in 3 Appendices. In the 3rd appendix we find the SOPs divided into 15 general, 15 laboratory, and 36 clinical. 
The aim of this article is to propose the SOPs of emergencies that can occur, since they have not been already established by AIFA. We have developed and structured them to highlight for each one the chain of responsibilities: drafting, verification and approval with the date of issue and any subsequent revisions.

Obviously the number of SOPs for a Phase I trial is high, but we report here only the those functional for an emergency occurrence.

\section{SOP 01}

Transfer of subjects and related medical information, in emergency situations, to the nearest hospital.

This SOP illustrates the methods of transferring a patient in emergency situations and the related information of the study in which he participates, which is admitted to a Phase I Unit located outside an hospital, without emergency facilities.

A collaboration agreement must be signed between the Phase I unit and the nearest hospital, which must be reachable in no more than 15 minutes and documented simulations must be carried out on a pre-established basis, to assess the critical factors (e.g. intense traffic and bad weather).

The agreement between parties will define:

- Methods of communication (which will take place via email) by the Phase I Unit, which will provide the hospital with all the necessary information on the study and IMP, including indication of any antidotes.

- Methods to share all the information relating to the study, through documented staff meetings, between the hospital representatives and the personnel involved.

- Methods of verification by the Director of Phase I who, by participating himself in staff meetings with contact persons and hospital staff, will be responsible for ensuring full understanding of the studies in progress and this will have to take place before enrollment of the first patient.

Access and transport of the patient to the hospital.

In case of emergency, the medical and nursing staff of the Unit, together with the Emergency Team, will stabilize the patient; in the event that, after stabilizing the patient, the emergency cannot be adequately managed, the physician and/or the resuscitator of Phase I:

- Contact the Hospital and announce the arrival of the patient at the DEA, providing all the information useful for the management of the case, relating to the trial in which the patient participates, including the need to use an antidote which, if necessary, is provided with information for correct use (as detailed in the Plan of Unforeseen Cases that always accompanies the patient);

- $\quad$ Organize the transfer by ambulance;

- A doctor of the CSEP goes to the DEA of the Hospital for advice as soon as possible, with an extract of the patient's medical record and other possible documentation, relative to the experimentation in which the patient participates, considered useful for the management of the case. Once the criticity has been overcome and stabilization is achieved, the patient returns to the CSEP following the overall clinical picture and in agreement with the DEA of the Hospital and/or other departments involved in the management of the case.

\section{SOP 03}

Training and maintenance of resuscitation procedures.

This SOP illustrates the planning of training, continuing education and the final assessment in the resuscitation procedures of clinical and non-clinical members working in the Phase I unit in order to make each person more and more competent in carrying out the emergency activities. The Quality Manager annually defines a census of the training needs of the personnel.

The Scientific Commission (SC) describes a programmatic planning of training activities for all interested personnel who will follow the courses provided by the company or by other bodies.

Basic training planning for initial resuscitation practices involving all personnel:

- Airway management - ventilation

- Intubation

- Administration of liquids

- Adrenaline administration

- $\quad$ CPR

- Use of the defibrillator

- $\mathrm{BLS} / \mathrm{D}$

In addition, medical personnel must also receive training in the immediate support of vital functions (ILS - Immediate Life Support) and in addition to Advanced Life Support if the unit is used to perform s.c. with high-risk drugs.

\section{Continuous training}

Medical staff

- UO Director: annual CPR and ALS

- $\quad$ Doctor (oncologist if applicable) and sub-investigator: Emergency updates (ILS) every 2 years, ALS every 2 years alternating with the previous one. 
Nursing staff

- $\quad$ Research nurses: BLSD every 2 years alternated with training on the management of critical cases and emergencies clinical emergencies.

- $\quad$ Nursing coordinator: BLSD every 2 years alternated with training on the management of critical cases and emergencies - clinical emergencies.

The various BLSD - ILS - ALS courses that train the personnel for the actions to be performed in the survival chain will be structured with duration, dedicated theoretical-practical time, ongoing evaluation and different final certification depending on the type of course in question and the relative documentation must be kept in the Phase I unit.

\section{SOP 08}

Randomization and breakdown codes preservation of the blind.

The purpose of this SOP is to describe the process that must be followed to

- $\quad$ Correct storage of trial randomization codes

- The blind collector's procedure in case of emergency

- Details on authorized personnel who will have access to the armored data.

The randomization procedure is performed and managed either centrally or by the Site Pharmacy with procedures specified in the study protocol and the treatment assigned to each patient is reported inside sealed envelopes and the PI is studied will ensure that the seals will be signed and dated. The envelopes are stored in the Site Pharmacy and can only be opened in case of emergency (e.g. antidote for the Investigational Medical Product (IMP) in the study in the case of the patient's Serious Adverse Event - SAE and/ or Suspected Unexpected Averse Serious Reaction - SUSAR).

The unmasking procedure must be declared in the study protocol and tested before the first patient is enrolled.

The PI must ensure that the process of breaking the blind in an emergency must be able to take place at any time and that all trial personnel are aware of the procedure.

Any member of the trial can request the break of the blind person in emergency and therefore the opening of the envelope relative to the patient, a procedure that must be authorized when possible by the study PI, documented and justified in detail in the Trial Master File, reporting the details of the subject involved, reason for the unmasking, name and role of those who request the unmasking and who performs it [6-8].

SOP 09: Communication procedure without delay of SUSAR and SAE of ongoing studies with the possibility of amending or interrupting the study.

This SOP illustrates the flow of information and the timing of the reporting of SAE and SUSAR of a serious nature by the Phase I Unit to the Sponsor and the competent bodies with the possibility to amend or interrupt the ongoing study without delay.

The notification of SAE and SUSAR is the ultimate responsibility of the Principal Investigator that uses the Council for International Organizations of Medical Sciences (CIOMS) module for SUSARs and a specific form indicated in the study protocol for SAEs.

The PI notifies the promoter within 24 hours of any SAE and/or SUSAR except those not subject to notification as indicated in the study protocol.

The recorded data will be available in real time and processed by the Sponsor's Pharmacovigilance unit. For suspected serious and unexpected adverse reactions that have been fatal or that have seriously endangered the subject, the promoter will ensure that all relevant information is notified by urgency to the AIFA and the EC involved and in any case within 7 calendar days from when the promoter became aware of it; for all other adverse reactions the promoter has 15 days from when he became aware of them, to communicate them to the Ministry of Health and to the EC involved.

The promoter also informs the other investigators.

\section{Conclusions}

According to the latest update published on the AIFA website of 03/14/2019, the Phase I centers certificated in Italy are 135 In 2018 the AIFA report concerning the clinical trials of 2017 was presented from which it appears that the 809/2015 determination certainly contributed to the increase in Phase I studies in Italy. In fact, the percentage of applications for authorization to run Phase I studies is $14 \%$ of all clinical studies.

In previous years, this value was much lower, respectively $10.3 \%$ in 2015 and $11.2 \%$ in 2016 (Fig). The increase in the number of structures certified for Phase I has contributed to increasing the number of studies conducted, although in Italy there is a scarce predisposition, from healthy volunteers and/or patients, to partici- 
pate in this type of studies given the commitments required. The reasons that explain the low percentages of Phase I studies in Italy, despite the increase in certified centers, are to be found in the perhaps excessive bureaucratization of the authorization process that slows down procedures compared to the rest of Europe and with high fees to pay to Regulatory Authorities and Ethics Committees charged to the Sponsors.

Italy, with this process of self-certification, has contributed to increasing the quality of studies also through the ad hoc Standard Operating Procedures that each center will have to adopt and I have developed these SOPs as a possible contribution to a further increase in quality.

All this should lead to greater attractiveness of our country in the eyes of foreign sponsors by offering them high quality clinical research and thus increasing our competitiveness with the rest of Europe.

\section{Bibliography}

1. Declaration of Helsinki. Ethical Principles for Medical Research Involving Human Subjects (1964).

2. Good Clinical Practice (GCP) for trials on medical products in the European Community" (1996). Decreto Ministeriale del 15 luglio. Recepimento delle linee guida della U. E. di Buona Pratica Clinica per la esecuzione delle sperimentazioni cliniche dei medicinali (1997).

3. http://www.agenziafarmaco.gov.it/sites/default/files/

4. http://www.agenziafarmaco.gov.it/sites/default/files/GUDetermina_451-2016+allegato.pdf

5. http://www.agenziafarmaco.gov.it/sites/default/ files/2019_07_11_Elenco-Strutture-Fase I.pdf

6. Statutory Instrument 2004/1031: The Medicines for Human Use (Clinical Trials) Regulations (2004).

7. Statutory Instrument 2006/1928: The Medicines for Human Use (Clinical Trials) Amendment Regulations (2006).

8. ICH Topic E6 (R2) Guideline for Good Clinical Practice.

Volume 3 Issue 12 December 2019

(C) All rights are reserved by Daniela Maria Capuano and Roberto Verna. 